\title{
Noninvertible 1-form symmetry and Casimir scaling in 2D Yang-Mills theory
}

\author{
Mendel Nguyen, ${ }^{1, *}$ Yuya Tanizaki ${ }^{2, \dagger}$ and Mithat Ünsal ${ }^{1, \$}$ \\ ${ }^{1}$ Department of Physics, North Carolina State University, Raleigh, North Carolina 27607, USA \\ ${ }^{2}$ Yukawa Institute for Theoretical Physics, Kyoto University, Kyoto 606-8502, Japan
}

(Received 19 April 2021; accepted 26 July 2021; published 2 September 2021)

\begin{abstract}
Pure Yang-Mills theory in two spacetime dimensions shows exact Casimir scaling. Thus, there are infinitely many string tensions, and this has been understood as a result of nonpropagating gluons in two dimensions. From ordinary symmetry considerations, however, this richness in the spectrum of string tensions seems mysterious. Conventional wisdom has it that it is the center symmetry that classifies string tensions, but being finite it cannot explain infinitely many confining strings. In this paper, we resolve this discrepancy between dynamics and kinematics by pointing out the existence of a noninvertible 1-form symmetry, which is able to distinguish Wilson loops in different representations. We speculate on possible implications for Yang-Mills theories in three and four dimensions.
\end{abstract}

DOI: 10.1103/PhysRevD.104.065003

\section{INTRODUCTION}

In quantum gauge theories, string tensions are characteristic properties of confinement phenomena, as they specify the static quark-antiquark potentials. We can compute them theoretically as expectation values of Wilson loops for the gauge representation $\alpha$ of the test quark. For confining $\mathrm{SU}(N)$ Yang-Mills theory in three or four dimensions, it is expected that Wilson loops behave very differently over three different length scales (see Fig. 1) [1-8]:

(1) At short distances, the potential obeys Coulomb's law, with a coefficient given by the Casimir invariant $c_{\alpha}$.

(2) At intermediate distances, the potential becomes linear, with a string tension $T_{\alpha}$ depending on $\alpha$.

(3) At long distances, the potential remains linear, but the string tension $T_{\alpha}$ depends only on the $N$-ality of $\alpha$.

The last property can be understood as a result of string breaking via soft-gluon exchange. Moreover, we can nicely describe the relevant selection rule using the center symmetry $[9,10]$, or the 1-form symmetry [11,12]. However, this is not the whole story of confinement. In particular, the behavior at intermediate distances is curious: the theory is

\footnotetext{
*mendelnguyen@gmail.com

yuya.tanizaki@yukawa.kyoto-u.ac.jp

unsal.mithat@gmail.com
}

Published by the American Physical Society under the terms of the Creative Commons Attribution 4.0 International license. Further distribution of this work must maintain attribution to the author(s) and the published article's title, journal citation, and DOI. Funded by SCOAP ${ }^{3}$. already confining, but the string tensions are not characterized by center symmetry alone.

Driven by this curiosity, the authors were led in Ref. [13] to explore a similar phenomenon in a simpler confining gauge theory in three dimensions, where string tensions at any distance scale do not obey the $N$-ality rule. In that investigation, it was realized that a noninvertible 1-form symmetry is present in that model, and that it can distinguish Wilson loops in different representations even when their $N$-alities are the same. Thus, the following question naturally arises: does noninvertible 1-form symmetry also exist in Yang-Mills theory? If so, can it be used to classify various string tensions at intermediate distances?

In this paper, we consider properties of the confining strings of pure Yang-Mills theory in two spacetime

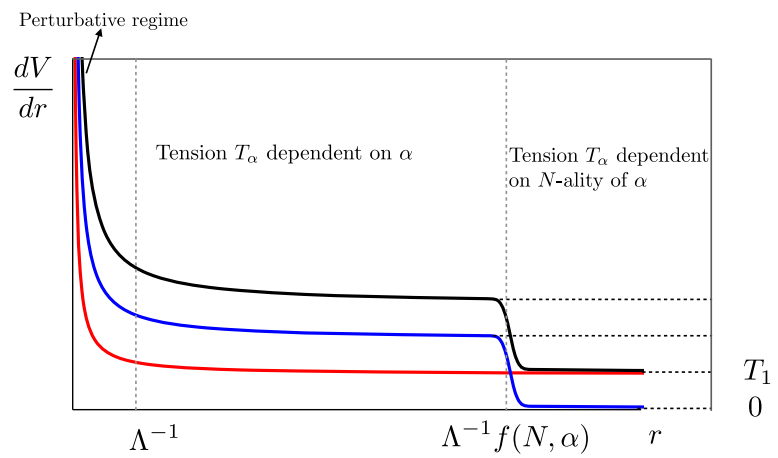

FIG. 1. Characteristic behavior of the derivative of the interquark potential in some representations $\alpha$ in $d=3,4 \mathrm{SU}(N)$ Yang-Mills theory. Here, we take the Young tableaux $\alpha=$ $(1,0, \ldots, 0),(1,0, \ldots, 0,1),(2,0, \ldots, 0,1)$ for the red, blue, and black curves, respectively. 
dimensions. It is well known that 2D Yang-Mills theory is exactly solvable [14,15], and the string tensions obey the Casimir scaling law, which says that the confining force is proportional to the Casimir invariant $c_{\alpha}$ of the test quark. In this case, we can understand from dynamical considerations why the string tensions need not be characterized by center symmetry. In two dimensions, there are no propagating gluons, and string breaking never occurs. Nevertheless, it behooves us to explain this phenomenon purely from considerations of symmetry, which must be an important step towards the harder cases of Yang-Mills theories in higher dimensions.

We find that there is in fact a good symmetry-based justification for the rich spectrum of confining strings in 2D Yang-Mills theory. After a brief review of the exact solution, we define a topological point-like disorder operator that can distinguish Wilson loops in different representations of the gauge group. At the end, we speculate on possible implications for the behavior of the confining strings of Yang-Mills theories in three and four dimensions at intermediate distances.

\section{2D YANG-MILLS AND CASIMIR SCALING}

Let us begin by reviewing some exact results in 2D YangMills theory $[14,15]$. Let $G$ be an arbitrary gauge group, which is assumed to be simple, connected, and simply connected.

Pure Yang-Mills theory on a spacetime $X$ with Riemannian metric $\mathrm{d} s^{2}=g_{i j} \mathrm{~d} x^{i} \otimes \mathrm{d} x^{j}$ is described by the action

$$
S=-\frac{1}{e^{2}} \int_{X} \operatorname{tr}(F \wedge * F),
$$

where $F$ is the field strength of the $G$ gauge field $A$,

$$
F=\mathrm{d} A+A \wedge A .
$$

A particularly special feature of the theory in two dimensions is its invariance under area-preserving diffeomorphisms. This becomes self-evident when we rewrite the action (1) in terms of the adjoint scalar $\phi=* F$ :

$$
S=-\frac{1}{e^{2}} \int_{X} \operatorname{tr}(\phi \wedge * \phi)=-\frac{1}{e^{2}} \int_{X} \operatorname{tr}\left(\phi^{2}\right) \sqrt{g} \mathrm{~d}^{2} x,
$$

where $g=\operatorname{det}\left(g_{i j}\right)$. Here, it should be noted that the metric $g_{i j}$ enters only through the area form $\sqrt{g} \mathrm{~d}^{2} x$.

As the only invariant of a top-degree form is its total integral, the partition function can depend on the metric $g_{i j}$ only through the total area $\mathcal{A}=\int_{X} \sqrt{g} \mathrm{~d}^{2} x$. Furthermore, since in two dimensions the gauge coupling $e$ has the dimensions of inverse length, the partition function is a function of the dimensionless combination $e^{2} \mathcal{A}$. In view of these considerations, we set $e=1$ and denote the partition function by $Z_{X}(\mathcal{A})$.

For our purposes, it is most useful to work with a particular lattice regularization known as the heat-kernel formulation, or the generalized Villain formulation [14,16$18]$. Let us denote links by $\ell, G$-valued link variables by $U_{\ell}$, plaquettes by $p$, and holonomies around $\partial p$ by $U_{p}:=\mathcal{P} \prod_{\ell \in \partial p} U_{\ell}$, where $\mathcal{P}$ denotes path ordering. Here, there is no restriction on the shapes of the plaquettes; they can be any polygon (e.g., triangles, squares, pentagons, etc.). The heat-kernel lattice formulation is defined by taking the one-plaquette weight to be

$$
Z_{\triangle}\left(U_{p}, \mathcal{A}_{p}\right)=\sum_{\alpha} d_{\alpha} \chi_{\alpha}\left(U_{p}\right) \exp \left(-c_{\alpha} \mathcal{A}_{p}\right),
$$

where $\alpha$ runs through unitary irreducible representations of $G, c_{\alpha}$ denotes the quadratic Casimir invariant of $\alpha, \chi_{\alpha}: G \rightarrow$ $\mathbb{C}$ is the character of $\alpha, d_{\alpha}=\chi_{\alpha}(\mathbb{I})$ is the dimension of $\alpha$, and $\mathcal{A}_{p}$ denotes the area of $p$. Here, $\mathbb{I} \in G$ is the identity element. The partition function is then given by

$$
Z_{X}(\mathcal{A})=\int \prod_{\ell} \mathrm{d} U_{\ell} \prod_{p} Z_{\triangle}\left(U_{p}, \mathcal{A}_{p}\right),
$$

where the link variables are integrated with respect to the normalized Haar measure of $G$.

In two spacetime dimensions, the heat-kernel formulation has a remarkable property: the partition function (5) is invariant under subdivisions. More precisely, if two plaquettes $p_{1}, p_{2}$ meet at a common link $\ell$, then one has the "sewing" property:

$$
\begin{gathered}
\int \mathrm{d} U_{\ell} Z_{\triangle}\left(U_{p_{1}}, \mathcal{A}_{p_{1}}\right) Z_{\triangle}\left(U_{p_{2}}, \mathcal{A}_{p_{2}}\right) \\
=Z_{\triangle}\left(U_{p_{1} \cup p_{2}}, \mathcal{A}_{p_{1}}+\mathcal{A}_{p_{2}}\right) .
\end{gathered}
$$

To see this, let us write the one-plaquette weights on the left-hand side explicitly as

$$
\begin{gathered}
Z_{\triangle}\left(U_{p_{1}}, \mathcal{A}_{p_{1}}\right)=\sum_{\alpha} d_{\alpha} \chi_{\alpha}\left(U_{1} U_{\ell}\right) \exp \left(-c_{\alpha} \mathcal{A}_{p_{1}}\right), \\
Z_{\triangle}\left(U_{p_{2}}, \mathcal{A}_{p_{2}}\right)=\sum_{\beta} d_{\beta} \chi_{\beta}\left(U_{\ell}^{-1} U_{2}\right) \exp \left(-c_{\alpha} \mathcal{A}_{p_{2}}\right),
\end{gathered}
$$

where we set $U_{p_{1}}=U_{1} U_{\ell}, \quad U_{p_{2}}=U_{\ell}^{-1} U_{2}$ so that $U_{p_{1} \cup p_{2}}=U_{1} U_{2}$. Using the following formula on characters,

$$
\int \mathrm{d} g \chi_{\alpha}(a g) \chi_{\beta}\left(g^{-1} b\right)=\delta_{\alpha, \beta} \frac{\chi_{\alpha}(a b)}{d_{\alpha}},
$$

we readily obtain Eq. (6). Therefore, this specific lattice formulation is already at the fixed point of the renormalization group, and reproduces the results of the continuum theory. 


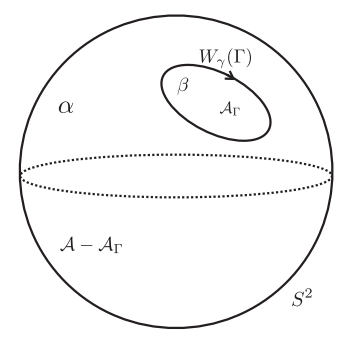

FIG. 2. Expectation value of a Wilson loop $W_{\gamma}(\Gamma)$ on $S^{2}$.

The partition function on any genus- $g$ spacetime $X=\Sigma_{g}$ is readily obtained as

$$
Z_{\Sigma_{g}}(\mathcal{A})=\sum_{\alpha} d_{\alpha}^{2-2 g} \exp \left(-c_{\alpha} \mathcal{A}\right)
$$

We note that, in the infinite area limit, which will give us the partition function on $\mathbb{R}^{2}$, we have

$$
Z_{\mathbb{R}^{2}}=Z_{\Sigma_{g}}(\mathcal{A} \rightarrow \infty)=1,
$$

as only the trivial representation $\alpha=\mathbf{1}$ contributes.

Let us now compute the expectation value of a single Wilson loop $W_{\gamma}(\Gamma)$ on $\mathbb{R}^{2}$. For convenience, we initially take $X=S^{2}$, and then take $\mathcal{A} \rightarrow \infty$ at the end while keeping the area $\mathcal{A}_{\Gamma}$ "enclosed" by $\Gamma$ held fixed (see Fig. 2). We have

$$
\begin{aligned}
\left\langle W_{\gamma}(\Gamma)\right\rangle_{S^{2}}= & \frac{1}{Z_{S^{2}}} \sum_{\alpha, \beta} d_{\alpha} d_{\beta} \mathrm{e}^{-c_{\alpha}\left(\mathcal{A}-\mathcal{A}_{\Gamma}\right)-c_{\beta} \mathcal{A}_{\Gamma}} \\
& \times \int \mathrm{d} U \chi_{\alpha}(U) \chi_{\gamma}(U) \chi_{\beta}\left(U^{-1}\right) .
\end{aligned}
$$

We then use another formula on characters,

$$
\int \mathrm{d} g \chi_{\alpha}(g) \chi_{\gamma}(g) \chi_{\beta}\left(g^{-1}\right)=N_{\alpha \gamma}^{\beta}
$$

where $N_{\alpha \gamma}^{\beta}$ is the multiplicity of $\beta$ in the decomposition of $\alpha \otimes \gamma$ into irreducible representations. This gives the Wilson loop average on $S^{2}$ as

$$
\left\langle W_{\gamma}(\Gamma)\right\rangle_{S^{2}}=\frac{1}{Z_{S^{2}}} \sum_{\alpha, \beta} N_{\alpha \gamma}^{\beta} d_{\alpha} d_{\beta} \mathrm{e}^{-c_{\alpha}\left(\mathcal{A}-\mathcal{A}_{\Gamma}\right)-c_{\beta} \mathcal{A}_{\Gamma}} .
$$

Taking the infinite area limit, only $\alpha=\mathbf{1}$ contributes as above, and as we clearly have $N_{\mathbf{1} \gamma}^{\beta}=\delta_{\beta, \gamma}$, it follows that the Wilson loop average on $\mathbb{R}^{2}$ is given by

$$
\left\langle W_{\gamma}(\Gamma)\right\rangle_{\mathbb{R}^{2}}=d_{\gamma} \exp \left(-c_{\gamma} \mathcal{A}_{\Gamma}\right) .
$$

Thus, Wilson loops in all representations obey area-law decay, and the string tensions are precisely dictated by Casimir scaling:

$$
T_{\gamma}=c_{\gamma}
$$

In particular, 2D pure Yang-Mills theory has infinitely many string tensions, which cannot be solely characterized by the center symmetry, or " $N$-ality." Is there any kinematical way to understand this result?

\section{NONINVERTIBLE 1-FORM SYMMETRY}

For a spacetime point $x$ and a conjugacy class $[U]=$ $\left\{g U g^{-1} \mid g \in G\right\}$ of $G$, we define a disorder operator $V_{[U]}(x)$ by the following injunction:

Delete the point $x$ from spacetime, and perform the path integral over gauge fields A with holonomy $\operatorname{hol}_{C}(A) \in[U]$ for small clockwise-oriented circles $C$ surrounding $x$.

This is a 2D version of Gukov-Witten surface operators in 4D gauge theories $[19,20]$. We note that this operator is gauge invariant, as we fix the conjugacy class of the holonomy instead of the holonomy itself. Furthermore, this operator is topological thanks to the invariance under area-preserving diffeomorphisms. But as we will see by explicit calculation, this operator does not necessarily have an inverse. Hence, we must view $V_{[U]}(x)$ as the generator of a noninvertible 1-form symmetry.

From a modern perspective on symmetry in relativistic field theory, a conservation law is interpreted as the existence of topological operators [11]. Noninvertible symmetry is a new kind of symmetry based on this idea, but the requirement that the symmetry elements obey a group-like multiplication law is relaxed. So far, the utility of this notion has been demonstrated mainly in the context of 2D field theories [21-28]. ${ }^{1}$ Our operator $V_{[U]}(x)$ has an analogous property, but it acts on line operators instead of point-like operators.

Using the heat-kernel lattice formulation, we can easily compute correlation functions of these disorder operators. All one needs to do is to pick out an infinitesimal plaquette $p$ containing the point $x$ of the dual lattice and a representative $U$ of the conjugacy class $[U]$, and then fix the path-ordered product of link variables $U_{p}$ to be $U^{-1}$.

For example, the $n$-point function of the disorder operators on $S^{2}$ can be computed $a^{2}$

$$
\left\langle\prod_{i=1}^{n} V_{\left[U_{i}\right]}\left(x_{i}\right)\right\rangle_{S^{2}}=\frac{1}{Z_{S^{2}}} \sum_{\alpha} d_{\alpha}^{2} \mathrm{e}^{-c_{\alpha} \mathcal{A}} \prod_{i=1}^{n} \frac{\chi_{\alpha}\left(U_{i}\right)}{d_{\alpha}} .
$$

\footnotetext{
${ }^{1}$ For applications to 3D gauge theories, see Refs. [13,29].

${ }^{2}$ We note that in the axiomatic approach to 2D YangMills theory, the two- and three-point functions here are precisely the "cylinder" and "pants" amplitudes from which all other amplitudes are built according to the general cutting-and-gluing law [15].
} 


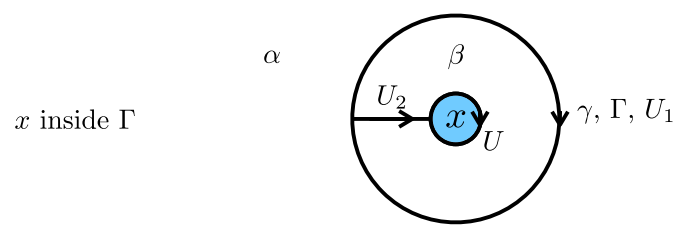

$x$ outside $\Gamma$

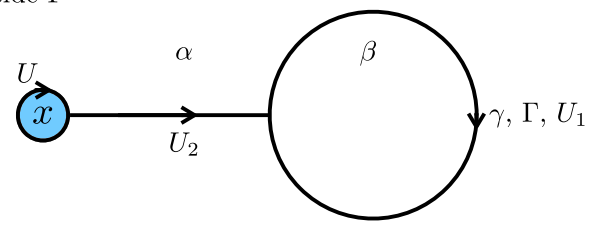

FIG. 3. Defect operator $V_{[U]}(x)$ inside and outside of the Wilson loop $W_{\gamma}(\Gamma)$.

In particular, the locations of the insertion points $x_{i}$ do not appear in the vacuum expectation values, which confirms that these operators are topological.

For us, the important thing about the disorder operators $V_{[U]}(x)$ is that they act nontrivially on Wilson loops. What is more, they can distinguish Wilson loops in different representations. In particular, on $\mathbb{R}^{2}$, we have

$\left\langle W_{\gamma}(\Gamma) V_{[U]}(x)\right\rangle= \begin{cases}\frac{\chi_{\gamma}(U)}{d_{\gamma}}\left\langle W_{\gamma}(\Gamma)\right\rangle & \text { for } x \text { inside } \Gamma, \\ \left\langle W_{\gamma}(\Gamma)\right\rangle & \text { for } x \text { outside } \Gamma .\end{cases}$

Let us point out that the noninvertible 1-form symmetry generated by the $V_{[U]}(x)$ actually contains the 1 -form center symmetry as a special case, which is similar to the 3D semiAbelian theory [13]. Namely, the 1-form center symmetry is generated by the $V_{[U]}(x)$ with $U$ in the center of $G$. For $G=\mathrm{SU}(N)$, the center elements can be written as $U=\omega \mathbb{I}$ with $\omega^{N}=1$, and we have

$$
\frac{\chi_{\gamma}(\omega \mathbb{I})}{d_{\gamma}}=\omega^{|\gamma|},
$$

where $|\gamma|$ is the $N$-ality of $\gamma$. Thus, for this specific choice, $V_{[\omega \mathrm{II}]}(x)$ is invertible.

Let us now prove Eq. (18). As before, we initially work on $S^{2}$ and take the infinite area limit at the end. Consider first the case where $x$ is inside $\Gamma$. According to Fig. 3, we get

$$
\begin{aligned}
& \left\langle W_{\gamma}(\Gamma) V_{[U]}(x)\right\rangle_{S^{2}} \\
& =\frac{1}{Z_{S^{2}}} \sum_{\alpha, \beta} d_{\alpha} d_{\beta} \exp \left(-c_{\alpha}\left(\mathcal{A}-\mathcal{A}_{\Gamma}\right)-c_{\beta} \mathcal{A}_{\Gamma}\right) \\
& \quad \times \int \mathrm{d} U_{1} \mathrm{~d} U_{2} \chi_{\alpha}\left(U_{1}\right) \chi_{\gamma}\left(U_{1}\right) \chi_{\beta}\left(U_{2} U_{1}^{-1} U_{2}^{-1} U\right) .
\end{aligned}
$$

We can easily evaluate the group integrals with the help of yet another formula on characters,

$$
\int \mathrm{d} g \chi_{\alpha}\left(g a g^{-1} b\right)=\frac{\chi_{\alpha}(a) \chi_{\alpha}(b)}{d_{\alpha}}
$$

together with Eq. (13). Then, Eq. (20) becomes

$$
\begin{aligned}
& \left\langle W_{\gamma}(\Gamma) V_{[U]}(x)\right\rangle_{S^{2}} \\
& \quad=\frac{1}{Z_{S^{2}}} \sum_{\alpha, \beta} N_{\alpha \gamma}^{\beta} d_{\alpha} \chi_{\beta}(U) \mathrm{e}^{-c_{\alpha}\left(\mathcal{A}-\mathcal{A}_{\Gamma}\right)-c_{\beta} \mathcal{A}_{\Gamma}} .
\end{aligned}
$$

Now taking the $\mathcal{A} \rightarrow \infty$ limit, this becomes

$$
\left\langle W_{\gamma}(\Gamma) V_{[U]}(x)\right\rangle=\chi_{\gamma}(U) \exp \left(-c_{\gamma} \mathcal{A}_{\Gamma}\right) .
$$

Comparison with Eq. (15) gives the first half of Eq. (18). Now consider the case where $x$ is outside of $\Gamma$. Then,

$$
\begin{aligned}
& \left\langle W_{\gamma}(\Gamma) V_{[U]}(x)\right\rangle_{S^{2}} \\
& =\frac{1}{Z_{S^{2}}} \sum_{\alpha, \beta} d_{\alpha} d_{\beta} \exp \left(-c_{\alpha}\left(\mathcal{A}-\mathcal{A}_{\Gamma}\right)-c_{\beta} \mathcal{A}_{\Gamma}\right) \\
& \quad \times \int \mathrm{d} U_{1} \mathrm{~d} U_{2} \chi_{\alpha}\left(U_{2} U U_{2}^{-1} U_{1}\right) \chi_{\gamma}\left(U_{1}\right) \chi_{\beta}\left(U_{1}^{-1}\right) .
\end{aligned}
$$

We evaluate the group integrals as before using Eqs. (21) and (13), obtaining

$$
\left\langle W_{\gamma}(\Gamma) V_{[U]}(x)\right\rangle_{S^{2}}=\frac{1}{Z_{S^{2}}} \sum_{\alpha, \beta} N_{\alpha \gamma}^{\beta} \chi_{\alpha}(U) d_{\beta} \mathrm{e}^{-c_{\alpha}\left(\mathcal{A}-\mathcal{A}_{\Gamma}\right)-c_{\beta} \mathcal{A}_{\Gamma}} .
$$

Taking $\mathcal{A} \rightarrow \infty$, this becomes

$$
\left\langle W_{\gamma}(\Gamma) V_{[U]}(x)\right\rangle=d_{\gamma} \exp \left(-c_{\gamma} \mathcal{A}_{\Gamma}\right) .
$$

We now get the second half of Eq. (18), which completes the proof.

This result (18) shows that we can measure the representation of the Wilson loop by using the topological defect operator $V_{[U]}$. In order to see this, let us rephrase this result in terms of canonical quantization on $S^{1} \times \mathbb{R}_{\text {time }}$. Performing the canonical quantization in the temporal gauge, an eigenstate wave function is given by a Wilson loop with some irreducible representation $\alpha$ wrapping $S^{1}$, and let us denote it as $|\alpha\rangle$. Then, Eq. (18) gives

$$
V_{[U]}(x)|\alpha\rangle=\frac{\chi_{\alpha}(U)}{d_{\alpha}}|\alpha\rangle .
$$

This tells that, using the local operator $V_{[U]}$, we can construct the projection operator onto a specific state as $|\alpha\rangle\langle\alpha|=d_{\alpha} \int \mathrm{d} g \chi_{\alpha}\left(g^{-1}\right) V_{[g]}(x){ }^{3}$

\footnotetext{
${ }^{3}$ This shows that the Hilbert space for 2D Yang-Mills theory decomposes into distinct sectors labeled by $\alpha$. It has been known that such a decomposition occurs with conventional $(d-1)$-form symmetry in $d$ spacetime dimensions [30-32]. In this viewpoint, we have found that noninvertible 1-form symmetry also decomposes the Hilbert space.
} 
As an example, consider the case $G=\mathrm{SU}(2)$, and let us detect its adjoint test quark. The center symmetry, $V_{[-\mathbb{I}]}$, does not detect it because the adjoint representation has trivial $N$-ality. On the other hand, if we choose $U=\mathrm{i} \sigma_{3}$ for instance, then we find

$$
\frac{\chi_{\text {adj }}\left(\mathrm{i} \sigma_{3}\right)}{d_{\text {adj }}}=-\frac{1}{3} \neq 1
$$

Therefore, $V_{[U]}$ can distinguish the adjoint string from the trivial one, and it allows us to explain the linear confinement of adjoint quarks very naturally. We also note that this operator is not invertible: by acting on the fundamental Wilson loop, we have

$$
\frac{\chi_{\mathrm{fd}}\left(\mathrm{i} \sigma_{3}\right)}{d_{\mathrm{fd}}}=0,
$$

and thus the inverse element cannot exist. In this way, the noninvertible topological operators $V_{[U]}$ successfully explain the violation of the $N$-ality rule in 2D YangMills theory from the viewpoint of symmetry.

\section{SUMMARY AND DISCUSSION}

In this work, we considered the question of why Casimir scaling should be exact in 2D Yang-Mills theory. This has been understood as a result of dynamics, as gluons in two dimensions do not propagate. However, it was not known if it could be understood from symmetry. The conventional center symmetry cannot explain why such a selection rule can exist. We have resolved this discrepancy by showing the existence of noninvertible 1-form symmetry generated by the defect operator $V_{[U]}(x)$.

This success for 2D Yang-Mills theory is encouraging for the prospect of a similar thing happening in three and four dimensions. For 3D Yang-Mills theory, the groundstate wave functional has been well studied numerically in Ref. [33], based on theoretical proposals in Refs. [34,35]. There, it was observed that the wave functional is proportional to the Boltzmann weight of 2D Yang-Mills theory at long distances. This "dimensional reduction" is supposed to be relevant to explain Casimir scaling at intermediate distances in higher dimensions, and it may give us a good hint for extending our study in two dimensions to higher dimensions.

At the same time, the $N$-ality rule should set in at large enough distances, so it seems that the noninvertible symmetry cannot be exact in three or four dimensions. While this is actually correct at finite $N$, we can still be optimistic in the $N=\infty$ theory. In the large- $N$ limit, the factorization theorem tells us that, for example,

$$
\left\langle W_{\mathrm{adj}}(C)\right\rangle=\left|\left\langle W_{\mathrm{fd}}(C)\right\rangle\right|^{2}+O\left(N^{-2}\right)
$$

Therefore, at $N=\infty$, the adjoint confining string never breaks, and its tension must be twice as large as that of the fundamental string. It is an interesting problem for the future to determine whether this can be interpreted as a result of (large- $N$ emergent) noninvertible 1-form symmetry.

\section{ACKNOWLEDGMENTS}

The authors thank Jeff Greensite, Zohar Komargodski, and Sahand Seifnashri for a useful discussion. The work of Y. T. was partially supported by JSPS KAKENHI Grant-inAid for Research Activity Start-up, 20K22350. M. Ü. acknowledges support from U.S. Department of Energy, Office of Science, Office of Nuclear Physics under Award No. DE-FG02-03ER41260.
[1] J. Ambjorn, P. Olesen, and C. Peterson, Stochastic confinement and dimensional reduction. 2. Threedimensional SU(2) lattice gauge theory, Nucl. Phys. B240, 533 (1984).

[2] G. I. Poulis and H. D. Trottier, 'Gluelump' spectrum and adjoint source potential in lattice QCD in three-dimensions, Phys. Lett. B 400, 358 (1997).

[3] G. S. Bali, Casimir scaling of SU(3) static potentials, Phys. Rev. D 62, 114503 (2000).

[4] O. Philipsen and H. Wittig, String breaking in SU(2) YangMills theory with adjoint sources, Phys. Lett. B 451, 146 (1999).

[5] P. Stephenson, Breaking of the adjoint string in $(2+1)$ dimensions, Nucl. Phys. B550, 427 (1999).
[6] P. de Forcrand and O. Philipsen, Adjoint string breaking in 4D SU(2) Yang-Mills theory, Phys. Lett. B 475, 280 (2000).

[7] J. Greensite, The confinement problem in lattice gauge theory, Prog. Part. Nucl. Phys. 51, 1 (2003).

[8] B. H. Wellegehausen, A. Wipf, and C. Wozar, Casimir scaling and string breaking in G(2) gluodynamics, Phys. Rev. D 83, 016001 (2011).

[9] G. 't Hooft, On the phase transition towards permanent quark confinement, Nucl. Phys. B138, 1 (1978).

[10] A. M. Polyakov, Thermal properties of gauge fields and quark liberation, Phys. Lett. 72B, 477 (1978).

[11] D. Gaiotto, A. Kapustin, N. Seiberg, and B. Willett, Generalized global symmetries, J. High Energy Phys. 02 (2015) 172. 
[12] E. Sharpe, Notes on generalized global symmetries in QFT, Fortschr. Phys. 63, 659 (2015).

[13] M. Nguyen, Y. Tanizaki, and M. Ünsal, Semi-Abelian gauge theories, non-invertible symmetries, and string tensions beyond $N$-ality, J. High Energy Phys. 03 (2021) 238.

[14] A. A. Migdal, Recursion equations in gauge theories, Sov. Phys. JETP 42, 413 (1975).

[15] E. Witten, On quantum gauge theories in two dimensions, Commun. Math. Phys. 141, 153 (1991).

[16] J. M. Drouffe, Transitions and duality in gauge lattice systems, Phys. Rev. D 18, 1174 (1978).

[17] P. Menotti and E. Onofri, The action of $\mathrm{SU}(N)$ lattice gauge theory in terms of the heat kernel on the group manifold, Nucl. Phys. B190, 288 (1981).

[18] C. B. Lang, C. Rebbi, P. Salomonson, and B. S. Skagerstam, The transition from strong coupling to weak coupling in the SU(2) lattice gauge theory, Phys. Lett. 101B, 173 (1981).

[19] S. Gukov and E. Witten, Rigid surface operators, Adv. Theor. Math. Phys. 14, 87 (2010).

[20] S. Gukov, Surface operators, arXiv:1412.7127.

[21] L. Bhardwaj and Y. Tachikawa, On finite symmetries and their gauging in two dimensions, J. High Energy Phys. 03 (2018) 189.

[22] M. Buican and A. Gromov, Anyonic chains, topological defects, and conformal field theory, Commun. Math. Phys. 356, 1017 (2017).

[23] D. S. Freed and C. Teleman, Topological dualities in the Ising model, arXiv:1806.00008.

[24] C.-M. Chang, Y.-H. Lin, S.-H. Shao, Y. Wang, and X. Yin, Topological defect lines and renormalization group flows in two dimensions, J. High Energy Phys. 01 (2019) 026.
[25] R. Thorngren and Y. Wang, Fusion category symmetry I: Anomaly in-flow and gapped phases, arXiv:1912.02817.

[26] W. Ji and X.-G. Wen, Categorical symmetry and noninvertible anomaly in symmetry-breaking and topological phase transitions, Phys. Rev. Res. 2, 033417 (2020).

[27] Z. Komargodski, K. Ohmori, K. Roumpedakis, and S. Seifnashri, Symmetries and strings of adjoint $\mathrm{QCD}_{2}$, J. High Energy Phys. 03 (2021) 103.

[28] D. Aasen, P. Fendley, and R. S. Mong, Topological defects on the lattice: Dualities and degeneracies, arXiv:2008 .08598 .

[29] T. Rudelius and S.-H. Shao, Topological operators and completeness of spectrum in discrete gauge theories, J. High Energy Phys. 12 (2020) 172.

[30] S. Hellerman and E. Sharpe, Sums over topological sectors and quantization of Fayet-Iliopoulos parameters, Adv. Theor. Math. Phys. 15, 1141 (2011).

[31] E. Sharpe, Undoing decomposition, Int. J. Mod. Phys. A 34, 1950233 (2019).

[32] Y. Tanizaki and M. Unsal, Modified instanton sum in QCD and higher-groups, J. High Energy Phys. 03 (2020) 123.

[33] J. Greensite, H. Matevosyan, S. Olejnik, M. Quandt, H. Reinhardt, and A. P. Szczepaniak, Testing proposals for the Yang-Mills vacuum wavefunctional by measurement of the vacuum, Phys. Rev. D 83, 114509 (2011).

[34] J. Greensite and S. Olejnik, Dimensional reduction and the Yang-Mills vacuum state in $2+1$ dimensions, Phys. Rev. D 77, 065003 (2008).

[35] D. Karabali, C.-j. Kim, and V. P. Nair, On the vacuum wave function and string tension of Yang-Mills theories in $(2+1)$-dimensions, Phys. Lett. B 434, 103 (1998). 